\title{
Normal Families and Growth of Meromorphic Functions with Their Kth Derivatives
}

\author{
Jianming $Q i \mathbb{D},{ }^{1,2}$ Fanning Meng $\mathbb{D},{ }^{3}$ and Wenjun Yuan $\mathbb{D}^{3}$ \\ ${ }^{1}$ Liberal Arts College/Business College, Shanghai Dianji University, Shanghai 201306, China \\ ${ }^{2}$ Department of Physics and Mathematics, University of Eastern Finland, 80101 Joensuu, Finland \\ ${ }^{3}$ School of Mathematics and Information Science, Guangzhou University, Guangzhou 510006, China \\ Correspondence should be addressed to Jianming Qi; qijianmingdaxia@163.com and Fanning Meng; mfnfdbx@163.com
}

Received 6 November 2017; Accepted 12 April 2018; Published 3 June 2018

Academic Editor: Hugo Leiva

Copyright ( 2018 Jianming Qi et al. This is an open access article distributed under the Creative Commons Attribution License, which permits unrestricted use, distribution, and reproduction in any medium, provided the original work is properly cited.

Relying on the normal family theory, we mainly study uniqueness problems of meromorphic functions and their $k$ th derivatives and estimate sharply the growth order of their meromorphic functions. Our theorems improve some previous results.

\section{Introduction and Main Results}

In this paper, a meromorphic function means a function that is meromorphic in the whole complex plane $\mathbb{C}$. We will use the fundamental results and the standard notation of the Nevanlinna theory of meromorphic functions such as $T(r, f), m(r, f)$, and $N(r, f)$ as explained in [1].

Let $\mathfrak{R}$ be a rational function (or a polynomial) that belongs asymptotically to $k r^{\alpha}$ as $r \rightarrow \infty$, where $k \neq 0, \alpha$ are constants. The degree of $\mathfrak{R}$ at infinity is defined as $\operatorname{deg} \mathfrak{R}:=$ $\operatorname{deg}_{\infty} \Re:=\max \{0, \alpha\}$.

Suppose that $f(z)$ and $g(z)$ are two nonconstant meromorphic functions. We define $a(z)$ as a meromorphic function or a finite complex number. In this paper, we define $g(z)-a(z)=0$ whenever $f(z)-a(z)=0$; then we write $f(z)=a(z) \Rightarrow g(z)=a(z)$. If $f(z)=a(z) \Rightarrow g(z)=a(z)$ and $g(z)=a(z) \Rightarrow f(z)=a(z)$, then we write $f(z)=$ $a(z) \Leftrightarrow g(z)=a(z)$ and say that $f(z)$ and $g(z)$ share $a(z) \mathrm{IM}$ (ignoring multiplicity). If $f-a$ and $g-a$ have the same zeros with the same multiplicities, we write $f(z)=a(z) \rightleftharpoons g(z)=$ $a(z)$ and say that $f$ and $g$ share $a \mathrm{CM}$ (counting multiplicity).

The subject on sharing values between entire functions and their derivatives was first studied by Rubel and Yang (cf. [2]). In 1977, they proved a result that if a nonconstant entire function $f$ and $f^{\prime}$ share two distinct finite numbers $a, b \mathrm{CM}$, then $f \equiv f^{\prime}$. Since then, sharing value problems have been studied by many authors and a number of profound results have been obtained (cf. $[1,3,4])$. To state our main results, we also need the following concepts.

Definition 1. The order $\rho(f)$ and the hyperorder $\rho_{2}(f)$ of a meromorphic function $f$ are both defined as follows:

$$
\begin{gathered}
\rho(f)=\limsup _{r \rightarrow \infty} \frac{\log T(r, f)}{\log r}, \\
\rho_{2}(f)=\limsup _{r \rightarrow \infty} \frac{\log \log T(r, f)}{\log r} .
\end{gathered}
$$

On estimating the order or hyperorder of meromorphic functions, there has been a lot of work on the growth order of meromorphic function to certain types of complex differential equations and complex difference equations (or complex functional equations) (cf. $[3,5-8])$.

In 2011, Lü and Yi ([9]) investigated the estimating order of meromorphic functions with their derivatives about sharing values and obtained the following results.

Theorem A. Let $f$ be a nonconstant meromorphic function with finitely many poles, and let $\alpha=Q_{1} e^{Q}$ and $\beta=Q_{2} e^{Q}$, where $Q_{1}, Q_{2}\left(\neq Q_{1}\right)$ and $Q$ are three polynomials. Let $k$ be $a$ 
positive integer. If all the zeros of $f-\alpha$ have multiplicity at least $k$ and

$$
\begin{aligned}
f(z) & =\alpha(z) \Longrightarrow \\
f^{(k)}(z) & =\alpha(z), \\
f(z) & =\beta(z) \Longleftrightarrow \\
f^{(k)}(z) & =\beta(z),
\end{aligned}
$$

then $\rho(f)<+\infty$.

Theorem B. Let $Q_{1}(\not \equiv 0)$ and $Q_{2}$ be two distinct polynomials, let $k$ be a positive integer, and let $f$ be a transcendental entire function with all the zeros of $f-Q_{2}$ having multiplicity at least k. If $f(z)=Q_{1}(z) \rightleftharpoons f^{(k)}(z)=Q_{1}(z)$ and $f(z)=Q_{2}(z) \Rightarrow$ $f^{(k)}(z)=Q_{2}(z)$, then one of the following cases must occur:

(1) $f=f^{(k)}$.

(2) $f(z)=Q_{2}(z)+A e^{\lambda z}$ and $(\mu-1) Q_{1}=\mu Q_{2}-Q_{2}^{(k)}$, where $A$ and $\lambda^{k}=\mu \neq 1$ are two nonzero constants.

In this paper, we continue to investigate the growth order and hyperorder of meromorphic functions with their derivatives about sharing functions and improve Theorems $A$ and B. Now, we state our results as follows.

Theorem 2. Let $f$ be a nonconstant meromorphic function with finitely many poles, and let $\alpha=R_{1} e^{\gamma}$ and $\beta=R_{2} e^{\gamma}$, where $R_{1}, R_{2}$ ( $\equiv R_{1}$ ) are two rational functions and $\gamma$ is an entire function. Let $k$ be a positive integer. If all the zeros of $f-\alpha$ have multiplicity at least $k$ and

$$
\begin{gathered}
f(z)=\alpha(z) \Longrightarrow \\
f^{(k)}(z)=\alpha(z), \\
f(z)=\beta(z) \Longleftrightarrow \\
f^{(k)}(z)=\beta(z),
\end{gathered}
$$

then $\rho_{2}(f) \leq \rho_{2}(\alpha)=\rho(\gamma)$.

Remark 3. The following examples show that the conclusion $\rho_{2}(f) \leq \rho(\gamma)$ is sharp.

Example 4. Let $f(z)=A e^{z}$, where $A$ is a nonzero constant. Let $\alpha(z)=e^{e^{-z}+z}$ and $\beta(z)=z e^{e^{-z}+z}$. Noting that $f=f^{(k)}$, one has

$$
\begin{gathered}
f(z)=\alpha(z) \Longrightarrow \\
f^{(k)}(z)=\alpha(z), \\
f(z)=\beta(z) \Longleftrightarrow \\
f^{(k)}(z)=\beta(z) .
\end{gathered}
$$

Thus, it satisfies the assumptions of Theorem 2 and $\rho_{2}(f)=$ $0<\rho_{2}(\alpha)=1$.
Example 5. Let $f(z)=z e^{z}+A e^{3 z}, \alpha(z)=z e^{z}$, and $\beta(z)=$ $(z-1 / 2) e^{z}$, where $A$ is a nonzero constant. Differentiating $f$ yields $f^{\prime}(z)=(z+1) e^{z}+3 A e^{3 z}$, and then $f(z)=\alpha(z) \Rightarrow$ $f^{\prime}(z)=\alpha(z)$ and $f(z)=\beta(z) \Leftrightarrow f^{\prime}(z)=\beta(z)$. Thus, $\rho_{2}(f) \leq$ $\rho_{2}(\alpha)$.

Theorem 6. Let $f$ be a nonconstant meromorphic function with finitely many poles, and let $\alpha=Q_{1} e^{Q}$ and $\beta=Q_{2} e^{Q}$, where $Q_{1}, Q_{2}\left(\not \equiv Q_{1}\right)$, and $Q$ are three polynomials with $\operatorname{deg} Q_{1}=q_{1}, \operatorname{deg} Q_{2}=q_{2}$, and $\operatorname{deg} Q=q$, where $q_{1}, q_{2}, q$ are three nonnegative integers. Let $k$ be a positive integer. If all the zeros of $f-\alpha$ have multiplicity at least $k$ and

$$
\begin{aligned}
f(z) & =\alpha(z) \Longrightarrow \\
f^{(k)}(z) & =\alpha(z), \\
f(z) & =\beta(z) \Longleftrightarrow \\
f^{(k)}(z) & =\beta(z),
\end{aligned}
$$

then $\rho(f) \leq \max \left\{q, q-\left(q_{2}-q_{1}\right) / k\right\}$.

Remark 7. The next two examples can illustrate that the conclusion of Theorem 6 is meaningful.

Example 8. Let $f(z)=z^{2} e^{z+1}+A e^{2 z}, \alpha(z)=z^{2} e^{z+1}$, and $\beta(z)=\left(z^{2}-(4 / 3) z-2 / 3\right) e^{z+1}$, where $A$ is a nonzero constant. Differentiating $f$ twice yields $f^{\prime}(z)=\left(z^{2}+2 z\right) e^{z+1}+2 A e^{2 z}$ and $f^{\prime \prime}(z)=\left(z^{2}+4 z+2\right) e^{z+1}+4 A e^{2 z}$, and then $f(z)=$ $\alpha(z) \Rightarrow f^{\prime \prime}(z)=\alpha(z)$ and $f(z)=\beta(z) \Leftrightarrow f^{\prime \prime}(z)=\beta(z)$. Thus, it satisfies the assumption of Theorem 6 . Noting that $k=2, \lambda=2$, and $\mu=4$, we obtain that $\rho(f)=1 \leq \max \{q=$ $\left.1, q-\left(q_{2}-q_{1}\right) / k=1-(2-2) / 2=1\right\}$ holds.

Example 9. Let $f(z)=z^{2} e^{z^{2}+1}+A e^{\sqrt{2} z}, \alpha(z)=z^{2} e^{z^{2}+1}$, and $\beta(z)=\left(-4 z^{4}-8 z^{2}-2\right) e^{z^{2}+1}$, where $A$ is a nonzero constant. Differentiating $f$ twice yields $f^{\prime}(z)=\left(2 z^{3}+2 z\right) e^{z^{2}+1}+$ $\sqrt{2} A e^{\sqrt{2} z}$ and $f^{\prime \prime}(z)=\left(4 z^{4}+10 z^{2}+2\right) e^{z^{2}+1}+2 A e^{\sqrt{2} z}$, and then $f(z)=\alpha(z) \Rightarrow f^{\prime \prime}(z)=\alpha(z)$ and $f(z)=\beta(z) \Leftrightarrow f^{\prime \prime}(z)=$ $\beta(z)$. Thus, it satisfies the assumption of Theorem 6 . We have $k=2, \lambda=\sqrt{2}, \mu=2$. We obtain that $\rho(f)=2 \leq \max \{q=$ $\left.2, q-\left(q_{2}-q_{1}\right) / k=2-(4-2) / 2=1\right\}$ holds.

Theorem 10. Let $f$ be a transcendental meromorphic function with finitely many poles, and let $\alpha=Q_{1} e^{Q}$ and $\beta=Q_{2} e^{Q}$, where $Q_{1}, Q_{2}\left(\neq Q_{1}\right)$, and $Q$ are three polynomials. Let $k$ be a positive integer. If all the zeros of $f-\alpha$ have multiplicity at least $k$ and $\rho(\beta)<\rho(f)$ and

$$
\begin{gathered}
f(z)=\alpha(z) \Longrightarrow \\
f^{(k)}(z)=\alpha(z), \\
f(z)=\beta(z) \rightleftharpoons \\
f^{(k)}(z)=\beta(z),
\end{gathered}
$$

then the conclusions of Theorem B still hold, and $Q(z)$ must be a constant. 
The next example will show if $f$ is rational; then Theorem 10 fails.

Example 11. Let $f(z)=z^{3}, Q_{2}(z)=(3 / 2) z^{3}-(3 / 2) z^{2}$, and $Q_{1}=Q=0$. Note that

$$
\begin{aligned}
\frac{f^{\prime}-Q_{2}}{f-Q_{2}} & =3, \\
f & =0 \Longrightarrow \\
f^{\prime} & =0 .
\end{aligned}
$$

However, it does not satisfy any case of Theorem B.

Remark 12. The condition $\rho(\beta)<\rho(f)$ plays an important pole in the proof of Theorem 10. Unfortunately, we do not know whether the condition could be weakened.

\section{Some Lemmas}

In order to prove our theorems, we need the following lemmas.

A family $\mathscr{F}$ of meromorphic functions defined on a domain $\Omega \subset \widehat{\mathbb{C}}$ (here $\widehat{\mathbb{C}}=\mathbb{C} \cup\{\infty\}$ ) is said to be normal in $\Omega$ if every sequence of elements of $\mathscr{F}$ contains a subsequence that converges locally uniformly in $\Omega$ with respect to the spherical metric to a meromorphic function or $\infty$ (cf. [10]).

Lemma 13 (see $[3,11])$. Let $\left\{f_{n}\right\}$ be a family of meromorphic (analytic) functions on the unit disc $\Delta$. If $a_{n} \rightarrow a,|a|<1$, and $f^{\sharp}\left(a_{n}\right) \rightarrow \infty$, then there exist

(a) a subsequence of $f_{n}$ (which we still write as $\left\{f_{n}\right\}$ );

(b) points $z_{n} \rightarrow z_{0},\left|z_{0}\right|<1$;

(c) positive numbers $\rho_{n} \rightarrow 0$,

such that $f_{n}\left(z_{n}+\rho_{n} \xi\right)=g_{n}(\xi) \rightarrow g(\xi)$ locally uniformly, where $g$ is a nonconstant meromorphic (entire) function on $\mathbb{C}$, such that

$$
\rho_{n} \leq \frac{M}{f_{n}^{\sharp}\left(a_{n}\right)},
$$

where $M$ is a constant that is independent of $n$.

Here, as usual, $f^{\sharp}(\xi)=\left|f^{\prime}(\xi)\right| /\left(1+|f(\xi)|^{2}\right)$ is the spherical derivative.

The next lemma is an extending result obtained by Lü and Qi in [12].

Lemma 14. Let $f$ be a meromorphic function of hyperorder $\rho_{2}(f)>0$. Then, for any $\epsilon>0$, there exists a sequence $z_{n} \rightarrow \infty$ such that

$$
f^{\sharp}\left(z_{n}\right)>e^{\left|z_{n}\right|^{d-\epsilon}}
$$

for large enough $n$, where $d=\rho_{2}(f)$ if $\rho_{2}(f)<\infty$ or $d$ is an arbitrary positive number.
Lemma 15 (cf. [5]). Let $f(z)$ be an entire function; $\rho(f)>1$. Then, for each $0<\mu<\rho(f)-1$, there exist points $a_{n} \rightarrow$ $\infty(n \rightarrow \infty)$, such that

$$
\lim _{n \rightarrow \infty} \frac{f^{\sharp}\left(a_{n}\right)}{\left|a_{n}\right|^{\mu}}=+\infty .
$$

Lemma 16 (cf. [1]). Suppose that $f(z)$ and $g(z)$ are two nonconstant meromorphic functions in the complex plane with $\rho(f)$ and $\rho(g)$ as their orders, respectively. Then

$$
\begin{aligned}
\rho(f g) & \leq \max \{\rho(f), \rho(g)\}, \\
\rho(f+g) & \leq \max \{\rho(f), \rho(g)\} .
\end{aligned}
$$

Lemma 17 (cf. [13]). Let $f(z)$ and $a(z)$ be meromorphic functions of finite order such that both of $f(z)$ and $a(z)$ have only finite many poles, $f(z)$ and $a(z)$ have no common poles, and the order of $a(z)$ is less than the order of $f(z)$. If $f(z)$ and $f^{(k)}(z)$ share $a(z) C M$, then $f^{(k)}(z)-a(z)=c(f(z)-a(z))$ for some nonzero constant $c$.

\section{The Proof of Theorem 2}

By the method in $[3,12,14-16]$, we will present our proof as follows.

Note that $\alpha=R_{1} e^{\gamma}$; then $\rho_{2}(\alpha)=\rho(\gamma)$. It suffices to show $\rho_{2}(f) \leq \rho(\gamma)$.

By reduction to absurdity, we assume that $\rho_{2}(f)>\rho(\gamma)$. Set $\rho_{2}(f)=d>c:=\rho(\gamma)$, and set $H:=f-\alpha$; then

(i) $H=0 \Rightarrow H^{(k)}=\alpha-\alpha^{(k)}$,

(ii) $H=\beta-\alpha \Leftrightarrow H^{(k)}=\beta-\alpha^{(k)}$,

where $\alpha^{(k)}=\phi e^{\gamma}$ and $\phi$ is a differential polynomial of $R_{1}$ and $\gamma$. Let

$$
\begin{aligned}
& \Upsilon=\beta-\alpha=\left(R_{2}-R_{1}\right) e^{\gamma}=: R e^{\gamma}, \\
& \theta=\beta-\alpha^{(k)}=\left(R_{2}-\phi\right) e^{\gamma}=: \Psi e^{\gamma} .
\end{aligned}
$$

We may set $F=H / \Upsilon$ and get $\rho_{2}(F)=\rho_{2}(f)=d$. By Lemma 14, for $0<\epsilon<(d-c) / 2$, there exists a sequence $w_{n} \rightarrow \infty$ as $n \rightarrow \infty$ such that

$$
F^{\sharp}\left(w_{n}\right)>e^{\left|w_{n}\right|^{\rho_{2}(F)-\epsilon}}=e^{\left|w_{n}\right|^{d-\epsilon}} .
$$

Note that $\Upsilon=\beta-\alpha=\left(R_{2}-R_{1}\right) e^{\gamma}$ has at most finitely many zeros and poles and $f-\alpha$ has at most finitely many poles. Then there exists a positive number $r$ such that $F$ has no poles in $D=\{z:|z|>r\}$.

In view of $w_{n} \rightarrow \infty$ as $n \rightarrow \infty$, without loss of generality, we assume that $\left|w_{n}\right| \geq r+1$ for all $n$. Define $D_{1}=\{z:|z|<1\}$ and

$$
F_{n}(z):=F\left(w_{n}+z\right)=\frac{H\left(w_{n}+z\right)}{\Upsilon\left(w_{n}+z\right)},
$$

and then every $F_{n}$ is analytic in $D_{1}$. Also $F_{n}^{\sharp}(0)=F^{\sharp}\left(w_{n}\right) \rightarrow$ $\infty$ as $n \rightarrow \infty$. It follows from Marty's criterion that $F_{n}$ is not normal at $z=0$. 
By Lemma 13 and choosing an appropriate subsequence of $F_{n}$ if necessary, we may assume that there exist sequences $z_{n}$ and $\rho_{n}$ with $\left|z_{n}\right|<r<1$ and $\rho_{n} \rightarrow 0$ such that the sequence $g_{n}$ is defined by

$$
g_{n}(\zeta)=F_{n}\left(z_{n}+\rho_{n} \zeta\right)=\frac{H\left(w_{n}+z_{n}+\rho_{n} \zeta\right)}{\Upsilon\left(w_{n}+z_{n}+\rho_{n} \zeta\right)} \longrightarrow g(\zeta)
$$

locally uniformly in $\mathbb{C}$, where $g$ is a nonconstant entire function of order at most 1 , whose zeros have multiplicity at least $k$ and

$$
\rho_{n} \leq \frac{M}{F_{n}^{\sharp}(0)}=\frac{M}{F^{\sharp}\left(w_{n}\right)} \leq M e^{-\left|w_{n}\right|^{d-\epsilon}}
$$

for a positive number $M$.

We assert that

$$
\rho_{n}^{k} \frac{H^{(k)}\left(w_{n}+z_{n}+\rho_{n} \zeta\right)}{\Upsilon\left(w_{n}+z_{n}+\rho_{n} \zeta\right)} \longrightarrow g^{(k)}(\zeta)
$$

We prove the assertion by induction. By (15), the assertion holds for $k=0$. We assume that the assertion is right for $k=l$; that is,

$$
\rho_{n}^{l} \frac{H^{(l)}\left(w_{n}+z_{n}+\rho_{n} \zeta\right)}{\Upsilon\left(w_{n}+z_{n}+\rho_{n} \zeta\right)} \longrightarrow g^{(l)}(\zeta)
$$

Now we will prove that the assertion is also right for $k=l+1$. Let

$$
G_{n}(\zeta)=\rho_{n}^{l} \frac{H^{(l)}\left(w_{n}+z_{n}+\rho_{n} \zeta\right)}{\Upsilon\left(w_{n}+z_{n}+\rho_{n} \zeta\right)}
$$

and then

$$
\begin{aligned}
G_{n}^{\prime}(\zeta) & \\
= & \rho_{n}^{l+1} \frac{H^{(l+1)}\left(w_{n}+z_{n}+\rho_{n} \zeta\right)}{\Upsilon\left(w_{n}+z_{n}+\rho_{n} \zeta\right)} \\
& -\rho_{n}^{l+1} \frac{H^{(l)}\left(w_{n}+z_{n}+\rho_{n} \zeta\right) \Upsilon^{\prime}\left(w_{n}+z_{n}+\rho_{n} \zeta\right)}{\Upsilon^{2}\left(w_{n}+z_{n}+\rho_{n} \zeta\right)} \\
= & \rho_{n}^{l+1} \frac{H^{(l+1)}\left(w_{n}+z_{n}+\rho_{n} \zeta\right)}{\Upsilon\left(w_{n}+z_{n}+\rho_{n} \zeta\right)} \\
& -\rho_{n} \frac{G_{n}(\zeta) \Upsilon^{\prime}\left(w_{n}+z_{n}+\rho_{n} \zeta\right)}{\Upsilon\left(w_{n}+z_{n}+\rho_{n} \zeta\right)} \longrightarrow g^{(l+1)}(\zeta) .
\end{aligned}
$$

Recall the above definition of degree of rational function; we have

$$
\begin{aligned}
\left|\frac{\Upsilon^{\prime}}{\Upsilon}\right|_{z=w_{n}+z_{n}+\rho_{n} \zeta} \mid & =\left|\frac{R^{\prime}+R \gamma^{\prime}}{R}\right|_{z=w_{n}+z_{n}+\rho_{n} \zeta} \mid \\
& \leq\left|w_{n}\right|^{q} M\left(\left|w_{n}+z_{n}+\rho_{n} \zeta\right|, \gamma^{\prime}\right) \\
& \leq\left|w_{n}\right|^{q} M\left(2\left|w_{n}\right|, \gamma^{\prime}\right) \\
& \leq\left|w_{n}\right|^{q} e^{A\left|w_{n}\right|^{c+e}},
\end{aligned}
$$

and here $A$ is a positive constant and $q$ is an integer. Noting that $0<\epsilon<(d-c) / 2$, we have $d-\epsilon>c+\epsilon$. Then, combining (16) and (21), we conclude that

$$
\left|\rho_{n} G_{n}(\zeta) \frac{\Upsilon^{\prime}\left(w_{n}+z_{n}+\rho_{n} \zeta\right)}{\Upsilon\left(w_{n}+z_{n}+\rho_{n} \zeta\right)}\right| \leq M\left|G_{n}(\zeta)\right|\left|w_{n}\right|^{q} e^{A\left|w_{n}\right|^{\mid c+\varepsilon}-\left|w_{n}\right|^{d-\epsilon}} \longrightarrow 0, \quad \text { as } n \longrightarrow \infty
$$

Then

$$
\rho_{n}^{l+1} \frac{H^{(l+1)}\left(w_{n}+z_{n}+\rho_{n} \zeta\right)}{\Upsilon\left(w_{n}+z_{n}+\rho_{n} \zeta\right)} \longrightarrow g^{(l+1)}(\zeta)
$$

Hence, we complete the proof of the assertion.

Obviously, $g^{(k)} \not \equiv 0$. Otherwise $g$ would be a polynomial of degree less than $k$ and could not have zeros of multiplicity at least $k$. In the following, we will prove that

(a) $g=0 \Rightarrow g^{(k)}=0$,

(b) $g=1 \Leftrightarrow g^{(k)}=0$.

Firstly, we prove (a). Suppose that $g\left(\zeta_{0}\right)=0$. By Hurwitz's theorem, there exists a sequence $\left(\zeta_{n}\right)_{n}, \zeta_{n} \rightarrow \zeta_{0}$, such that (for $n$ being sufficiently large)

$$
g_{n}\left(\zeta_{n}\right)=\frac{H\left(w_{n}+z_{n}+\rho_{n} \zeta_{n}\right)}{\Upsilon\left(w_{n}+z_{n}+\rho_{n} \zeta_{n}\right)}=0 .
$$

Thus, $H\left(w_{n}+z_{n}+\rho_{n} \zeta_{n}\right)=0$ and $H^{(k)}\left(w_{n}+z_{n}+\rho_{n} \zeta_{n}\right)=$ $\left(\alpha-\alpha^{(k)}\right)\left(w_{n}+z_{n}+\rho_{n} \zeta_{n}\right)=\left(R_{1}-\phi\right) e^{\gamma}\left(w_{n}+z_{n}+\rho_{n} \zeta_{n}\right)$.

By (16), (17), (21), and (22), we derive that

$$
\begin{aligned}
g^{(k)}\left(\zeta_{0}\right) & =\lim _{n \rightarrow \infty} \rho_{n}^{k} \frac{H^{(k)}\left(w_{n}+z_{n}+\rho_{n} \zeta_{n}\right)}{\Upsilon\left(w_{n}+z_{n}+\rho_{n} \zeta_{n}\right)} \\
& =\lim _{n \rightarrow \infty} \rho_{n}^{k} \frac{\left(R_{1}-\phi\right)\left(w_{n}+z_{n}+\rho_{n} \zeta_{n}\right)}{\left(R_{2}-R_{1}\right)\left(w_{n}+z_{n}+\rho_{n} \zeta_{n}\right)}=0 .
\end{aligned}
$$

Thus, $g(\zeta)=0 \Rightarrow g^{(k)}(\zeta)=0$. So (a) holds.

Similarly, we can prove $g=1 \Rightarrow g^{(k)}=0$. Now we prove

$$
\begin{aligned}
g^{(k)} & =0 \Longrightarrow \\
g & =1 .
\end{aligned}
$$


From (16) and (17), we have

$$
\rho_{n}^{k} \frac{H^{(k)}\left(w_{n}+z_{n}+\rho_{n} \zeta\right)-\theta\left(w_{n}+z_{n}+\rho_{n} \zeta\right)}{\Upsilon\left(w_{n}+z_{n}+\rho_{n} \zeta\right)} \longrightarrow g^{(k)}(\zeta)
$$

Suppose that $g^{(k)}\left(\eta_{0}\right)=0$. Then $g^{(k)} \neq \equiv 0$. Hence, by (27) and Hurwitz's theorem, there exists a sequence $\left(\eta_{n}\right)_{n}, \eta_{n} \rightarrow \eta_{0}$, such that (for $n$ being sufficiently large)

$$
H^{(k)}\left(w_{n}+z_{n}+\rho_{n} \eta_{n}\right)=\theta\left(w_{n}+z_{n}+\rho_{n} \eta_{n}\right)
$$

Then, it follows from the assumption that we get $H\left(w_{n}+z_{n}+\right.$ $\left.\rho_{n} \eta_{n}\right)=\Upsilon\left(w_{n}+z_{n}+\rho_{n} \eta_{n}\right)$. It implies that

$$
g\left(\eta_{0}\right)=\lim _{r \rightarrow \infty} \frac{H\left(w_{n}+z_{n}+\rho_{n} \eta_{n}\right)}{\Upsilon\left(w_{n}+z_{n}+\rho_{n} \eta_{n}\right)}=1
$$

Thus, we prove that $g^{(k)}=0 \Rightarrow g=1$ and obtain (b).

From (a) and (b), it is easy to deduce that $g \neq 0$. Thus, $g=$ $A e^{\lambda z}$; it contradicts with $g=1 \Rightarrow g^{(k)}=0$. So $\rho_{2}(f) \leq \rho(\gamma)$. Therefore, we complete the proof of Theorem 2 .

\section{The Proof of Theorem 6}

Using some ideas of $[3,14]$, we give the following proof of Theorem 6.

Let $H=f-\alpha$. Then all the zeros of $H$ have multiplicities at least $k$ and

$$
\begin{aligned}
H & =0 \Longrightarrow \\
H^{(k)} & =\alpha-\alpha^{(k)}, \\
H & =\beta-\alpha \Longleftrightarrow \\
H^{(k)} & =\beta-\alpha^{(k)},
\end{aligned}
$$

where $\alpha^{(k)}=P_{1} e^{\mathrm{Q}}$ and $P_{1}$ is a polynomial. A careful calculation leads us to obtain $\operatorname{deg} P_{1}=k \operatorname{deg} Q^{\prime}+\operatorname{deg} Q_{1}=$ $k(q-1)+q_{1}$. Let

$$
\begin{aligned}
& \gamma=\beta-\alpha=\left(Q_{2}-Q_{1}\right) e^{Q}:=P e^{Q}, \\
& \theta=\beta-\alpha^{(k)}=\left(Q_{2}-P_{1}\right) e^{Q}:=P_{2} e^{Q},
\end{aligned}
$$

where $P_{2}$ is a polynomial. Set $F=H / \gamma$.

If $\rho(F)>\max \left\{q, q-\left(q_{2}-q_{1}\right) / k\right\}$, by Lemma 15 , for each $0<\mu<\rho(F)-1$, there exist $w_{n} \rightarrow \infty$ such that, for $n \rightarrow \infty$,

$$
\lim _{n \rightarrow \infty} \frac{F^{\sharp}\left(w_{n}\right)}{\left|w_{n}\right|^{\mu}}=+\infty .
$$

In view of $w_{n} \rightarrow \infty$ as $n \rightarrow \infty$, without loss of generality, we may assume that $\left|w_{n}\right| \geq r+1$ for all $n$. Define $D_{1}=\{z:|z|<1\}$ and

$$
F_{n}(z):=F\left(w_{n}+z\right)=\frac{H\left(w_{n}+z\right)}{\gamma\left(w_{n}+z\right)}
$$

Note that $\gamma=\beta-\alpha=\left(Q_{2}-Q_{1}\right) e^{Q}$ has at most finitely many zeros and $f-\alpha$ has at most finitely poles; then there exists a positive number $r$ such that $F$ has no poles in $D=\{z:|z|>$ $r$, so $F_{n}(z)=F\left(w_{n}+z\right)$ is analytic in $D_{1}$. Therefore, all $F_{n}(z)$ are analytic in $D_{1}$. Thus, we have structured a family $\left(F_{n}\right)_{n}$ of holomorphic functions.

Also $F_{n}^{\sharp}(0)=F^{\sharp}\left(w_{n}\right) \rightarrow \infty$ as $n \rightarrow \infty$. It follows from Marty's criterion that $F_{n}$ is not normal at $z=0$. Therefore, by applying Lemma 13 and choosing an appropriate subsequence of $F_{n}$ if necessary, we may assume that there exist subsequences $z_{n}$ and $\rho_{n}$ with $\left|z_{n}\right|<r<1$ and $\rho_{n} \rightarrow 0$ such that the sequence $g_{n}$ is defined by

$$
g_{n}(\zeta):=F_{n}\left(z_{n}+\rho_{n} \zeta\right)=\frac{H\left(w_{n}+z_{n}+\rho_{n} \zeta\right)}{\gamma\left(w_{n}+z_{n}+\rho_{n} \zeta\right)} \longrightarrow g(\zeta)
$$

locally uniformly in $\mathbb{C}$, where $g$ is a nonconstant entire function of order at most 1 , whose zeros have multiplicity at least $k$ and

$$
\rho_{n} \leq \frac{M}{F_{n}^{\sharp}(0)}=\frac{M}{F^{\sharp}\left(w_{n}\right)} \leq M\left|w_{n}\right|^{-\mu}
$$

for a positive number $M$.

We assert that

$$
\rho_{n}^{k} \frac{H^{(k)}\left(w_{n}+z_{n}+\rho_{n} \zeta\right)}{\gamma\left(w_{n}+z_{n}+\rho_{n} \zeta\right)} \longrightarrow g^{(k)}(\zeta)
$$

We will use the mathematical induction to prove the assertion. By (34), the assertion is right for $k=0$. We assume that the assertion is right for $k=l$; that is,

$$
\rho_{n}{ }_{n} \frac{H^{(l)}\left(w_{n}+z_{n}+\rho_{n} \zeta\right)}{\gamma\left(w_{n}+z_{n}+\rho_{n} \zeta\right)} \longrightarrow g^{(l)}(\zeta)
$$


Now we prove that the assertion is also right when $k=l+1$. Let

$$
G_{n}(\zeta)=\rho_{n}^{l} \frac{H^{(l)}\left(w_{n}+z_{n}+\rho_{n} \zeta\right)}{\gamma\left(w_{n}+z_{n}+\rho_{n} \zeta\right)}
$$

and then

$$
\begin{aligned}
& G_{n}^{\prime}(\zeta) \\
& =\rho_{n}^{l+1} \frac{H^{(l+1)}\left(w_{n}+z_{n}+\rho_{n} \zeta\right)}{\gamma\left(w_{n}+z_{n}+\rho_{n} \zeta\right)} \\
& \quad-\rho_{n}^{l+1} \frac{H^{(l)}\left(w_{n}+z_{n}+\rho_{n} \zeta\right) \gamma^{\prime}\left(w_{n}+z_{n}+\rho_{n} \zeta\right)}{\gamma^{2}\left(w_{n}+z_{n}+\rho_{n} \zeta\right)} \\
& =\rho_{n}^{l+1} \frac{H^{(l+1)}\left(w_{n}+z_{n}+\rho_{n} \zeta\right)}{\gamma\left(w_{n}+z_{n}+\rho_{n} \zeta\right)} \\
& \quad-\rho_{n} \frac{G_{n}(\zeta) \gamma^{\prime}\left(w_{n}+z_{n}+\rho_{n} \zeta\right)}{\gamma\left(w_{n}+z_{n}+\rho_{n} \zeta\right)} \longrightarrow g^{(l+1)}(\zeta) .
\end{aligned}
$$

Since $\gamma=P e^{Q}$, we have

$$
\begin{aligned}
\rho_{n} & \frac{G_{n}(\zeta) \gamma^{\prime}\left(w_{n}+z_{n}+\rho_{n} \zeta\right)}{\gamma\left(w_{n}+z_{n}+\rho_{n} \zeta\right)} \\
& =\rho_{n} \frac{G_{n}(\zeta)\left(P^{\prime}+P Q^{\prime}\right)\left(w_{n}+z_{n}+\rho_{n} \zeta\right)}{P\left(w_{n}+z_{n}+\rho_{n} \zeta\right)} .
\end{aligned}
$$

In view of the degree of a polynomial function, one has

$$
\begin{aligned}
\left|\frac{\gamma^{\prime}}{\gamma}\right|_{z=w_{n}+z_{n}+\rho_{n} \zeta} \mid & =\left|\frac{P^{\prime}+P Q^{\prime}}{P}\right|_{z=w_{n}+z_{n}+\rho_{n} \zeta} \mid \\
& =\left|\left(\frac{P^{\prime}}{P}+Q^{\prime}\right)\right|_{z=w_{n}+z_{n}+\rho_{n} \zeta} \mid \\
& =O\left(\left|w_{n}\right|^{l_{1}}\right) \quad(\text { as } n \longrightarrow \infty),
\end{aligned}
$$

where $l_{1}:=\operatorname{deg} Q^{\prime}$ is a nonnegative integer. Then, combining (35) and (41) yields

$$
\left|\rho_{n} G_{n}(\zeta) \frac{\gamma^{\prime}\left(w_{n}+z_{n}+\rho_{n} \zeta\right)}{\gamma\left(w_{n}+z_{n}+\rho_{n} \zeta\right)}\right| \leq M\left|G_{n}(\zeta)\right|\left|w_{n}\right|^{l_{1}-\mu} \longrightarrow 0, \quad \text { as } n \longrightarrow \infty
$$

Furthermore,

$$
\rho_{n}^{l+1} \frac{H^{(l+1)}\left(w_{n}+z_{n}+\rho_{n} \zeta\right)}{\gamma\left(w_{n}+z_{n}+\rho_{n} \zeta\right)} \rightarrow g^{(l+1)}(\zeta)
$$

So we complete the proof of the assertion.

Obviously, $g^{(k)} \not \equiv 0$. Otherwise, $g$ would be a polynomial of degree less than $k$ and so could not have zeros of multiplicity at least $k$. In the following, we will prove

(c) $g=0 \Rightarrow g^{(k)}=0$,

(d) $g=1 \Leftrightarrow g^{(k)}=0$.

Firstly, we prove (c). Suppose that $g\left(\zeta_{0}\right)=0$. Then, by Hurwitz's theorem, we get that there exists a sequence $\left(\zeta_{n}\right)_{n}$, $\zeta_{n} \rightarrow \zeta_{0}$, such that (for $n$ being sufficiently large)

$$
g_{n}\left(\zeta_{n}\right)=\frac{H\left(w_{n}+z_{n}+\rho_{n} \zeta_{n}\right)}{\gamma\left(w_{n}+z_{n}+\rho_{n} \zeta_{n}\right)}=0
$$

Thus, $H\left(w_{n}+z_{n}+\rho_{n} \zeta_{n}\right)=0$ and $H^{(k)}\left(w_{n}+z_{n}+\rho_{n} \zeta_{n}\right)=$ $\left(Q_{1}-P_{1}\right) e^{Q}\left(w_{n}+z_{n}+\rho_{n} \zeta_{n}\right)$. By (36) and (39), we derive that

$$
\begin{aligned}
g^{(k)}\left(\zeta_{0}\right) & =\lim _{n \rightarrow \infty} \rho_{n}^{k} \frac{H^{(k)}\left(w_{n}+z_{n}+\rho_{n} \zeta_{n}\right)}{\gamma\left(w_{n}+z_{n}+\rho_{n} \zeta_{n}\right)} \\
& =\lim _{n \rightarrow \infty} \rho_{n}^{k} \frac{\left(Q_{1}-P_{1}\right)\left(w_{n}+z_{n}+\rho_{n} \zeta_{n}\right)}{P\left(w_{n}+z_{n}+\rho_{n} \zeta_{n}\right)} .
\end{aligned}
$$

Also,

$$
\begin{aligned}
\left|\frac{Q_{1}-P_{1}}{P}\right|_{z=w_{n}+z_{n}+\rho_{n} \zeta} \mid & =\left|\frac{Q_{1}-P_{1}}{Q_{2}-Q_{1}}\right|_{z=w_{n}+z_{n}+\rho_{n} \zeta} \mid \\
& =\left|\left(\frac{1-P_{1} / Q_{1}}{Q_{2} / Q_{1}-1}\right)\right|_{z=w_{n}+z_{n}+\rho_{n} \zeta} \mid \\
& =O\left(\left|w_{n}\right|^{l_{2}}\right), \quad(\text { as } n \longrightarrow \infty)
\end{aligned}
$$

where $l_{2}:=\max \left\{k(q-1)-\left(q_{2}-q_{1}\right), 0\right\}$ is a nonnegative integer. Then, combining (35) and (46) yields

$$
\left|\rho_{n}^{k} \frac{\left(Q_{1}-P_{1}\right)\left(w_{n}+z_{n}+\rho_{n} \zeta_{n}\right)}{P\left(w_{n}+z_{n}+\rho_{n} \zeta_{n}\right)}\right| \leq M^{k}\left|w_{n}\right|^{l_{2}-k \mu} \longrightarrow 0, \quad \text { as } n \longrightarrow \infty
$$


So,

$$
\begin{aligned}
g^{(k)}\left(\zeta_{0}\right) & =\lim _{n \rightarrow \infty} \rho_{n}^{k} \frac{H^{(k)}\left(w_{n}+z_{n}+\rho_{n} \zeta_{n}\right)}{\gamma\left(w_{n}+z_{n}+\rho_{n} \zeta_{n}\right)} \\
& =\lim _{n \rightarrow \infty} \rho_{n}^{k} \frac{\left(Q_{1}-P_{1}\right)\left(w_{n}+z_{n}+\rho_{n} \zeta_{n}\right)}{P\left(w_{n}+z_{n}+\rho_{n} \zeta_{n}\right)}=0 .
\end{aligned}
$$

Thus, $g(\zeta)=0 \Rightarrow g^{(k)}(\zeta)=0$ and (c) holds.

Next, we will prove that $g=1 \Rightarrow g^{(k)}=0$.

Similar to what was stated above, suppose that $g\left(\zeta_{0}\right)=$ 1. Then, by Hurwitz's theorem, there exists a sequence $\left(\zeta_{n}\right)_{n}$, $\zeta_{n} \rightarrow \zeta_{0}$, such that (for $n$ being sufficiently large)

$$
g_{n}\left(\zeta_{n}\right)=\frac{H\left(w_{n}+z_{n}+\rho_{n} \zeta_{n}\right)}{\gamma\left(w_{n}+z_{n}+\rho_{n} \zeta_{n}\right)}=1 .
$$

Thus, $H\left(w_{n}+z_{n}+\rho_{n} \zeta_{n}\right)=\gamma\left(w_{n}+z_{n}+\rho_{n} \zeta_{n}\right)$ and $H^{(k)}\left(w_{n}+\right.$ $\left.z_{n}+\rho_{n} \zeta_{n}\right)=\left(Q_{2}-P_{1}\right) e^{Q}\left(w_{n}+z_{n}+\rho_{n} \zeta_{n}\right)$. By (35) and (36), we deduce that

$$
\begin{aligned}
g^{(k)}\left(\zeta_{0}\right) & =\lim _{n \rightarrow \infty} \rho_{n}^{k} \frac{H^{(k)}\left(w_{n}+z_{n}+\rho_{n} \zeta_{n}\right)}{\gamma\left(w_{n}+z_{n}+\rho_{n} \zeta_{n}\right)} \\
& =\lim _{n \rightarrow \infty} \rho_{n}^{k} \frac{\left(Q_{2}-P_{1}\right)\left(w_{n}+z_{n}+\rho_{n} \zeta_{n}\right)}{\left(Q_{2}-Q_{1}\right)\left(w_{n}+z_{n}+\rho_{n} \zeta_{n}\right)} .
\end{aligned}
$$

By calculation,

$$
\begin{aligned}
& \left.\left|\frac{Q_{2}-P_{1}}{P}\right|_{z=w_{n}+z_{n}+\rho_{n} \zeta}|=| \frac{Q_{2}-P_{1}}{Q_{2}-Q_{1}}\right|_{z=w_{n}+z_{n}+\rho_{n} \zeta} \mid \\
& =\left|\left(\frac{Q_{2} / Q_{1}-P_{1} / Q_{1}}{Q_{2} / Q_{1}-1}\right)\right|_{z=w_{n}+z_{n}+\rho_{n} \zeta} \mid=O\left(\left|w_{n}\right|^{l_{3}}\right),
\end{aligned}
$$

$($ as $n \longrightarrow \infty)$

where $l_{3}:=\max \left\{k(q-1)-\left(q_{2}-q_{1}\right), 0\right\}=l_{2}$ is a nonnegative integer. Then, combining (35) and (51) shows that

$$
\left|\rho_{n}^{k} \frac{\left(Q_{2}-P_{1}\right)\left(w_{n}+z_{n}+\rho_{n} \zeta_{n}\right)}{P\left(w_{n}+z_{n}+\rho_{n} \zeta_{n}\right)}\right| \leq M^{k}\left|w_{n}\right|^{l_{3}-k \mu} \longrightarrow 0,
$$

as $n \longrightarrow \infty$.

So,

$$
\begin{aligned}
g^{(k)}\left(\zeta_{0}\right) & =\lim _{n \rightarrow \infty} \rho_{n}^{k} \frac{H^{(k)}\left(w_{n}+z_{n}+\rho_{n} \zeta_{n}\right)}{\gamma\left(w_{n}+z_{n}+\rho_{n} \zeta_{n}\right)} \\
& =\lim _{n \rightarrow \infty} \rho_{n}^{k} \frac{\left(Q_{2}-P_{1}\right)\left(w_{n}+z_{n}+\rho_{n} \zeta_{n}\right)}{P\left(w_{n}+z_{n}+\rho_{n} \zeta_{n}\right)}=0 .
\end{aligned}
$$

It implies that $g(\zeta)=1 \Rightarrow g^{(k)}(\zeta)=0$. Now, we prove

$$
\begin{aligned}
g^{(k)} & =0 \Longrightarrow \\
g & =1 .
\end{aligned}
$$

From (35), (36), (51), and (52), we have

$$
\rho_{n}^{k} \frac{H^{(k)}\left(w_{n}+z_{n}+\rho_{n} \zeta\right)-\theta\left(w_{n}+z_{n}+\rho_{n} \zeta\right)}{\gamma\left(w_{n}+z_{n}+\rho_{n} \zeta\right)} \longrightarrow g^{(k)}(\zeta) .
$$

Suppose that $g^{(k)}\left(\eta_{0}\right)=0$. Since $g^{(k)} \not \equiv 0$, by (55) and Hurwitz's theorem, there exists a sequence $\eta_{n}, \eta_{n} \rightarrow \eta_{0}$, such that (for $n$ being sufficiently large)

$$
H^{(k)}\left(w_{n}+z_{n}+\rho_{n} \eta_{n}\right)=\theta\left(w_{n}+z_{n}+\rho_{n} \eta_{n}\right) .
$$

By the assumption, we get $H\left(w_{n}+z_{n}+\rho_{n} \eta_{n}\right)=\gamma\left(w_{n}+z_{n}+\rho_{n} \eta_{n}\right)$ and

$$
g\left(\eta_{0}\right)=\lim _{r \rightarrow \infty} \frac{H\left(w_{n}+z_{n}+\rho_{n} \eta_{n}\right)}{\gamma\left(w_{n}+z_{n}+\rho_{n} \eta_{n}\right)}=1 .
$$

Thus, we prove $g^{(k)}=0 \Rightarrow g=1$ and obtain (d).

From (c) and (d), it is easy to deduce that $g \neq 0$. Thus, $g=A e^{\lambda z}$. But it contradicts with $g=1 \Rightarrow g^{(k)}=0$. So $\rho(F) \leq \max \left\{q, q-\left(q_{2}-q_{1}\right) / k\right\}$

Next, we will prove $\rho(f) \leq \rho(F) \leq \max \left\{q, q-\left(q_{2}-q_{1}\right) / k\right\}$.

If $\rho(\alpha)<\rho(f)$, noting the fact that $\rho(\beta-\alpha) \leq \rho(\alpha)$ and Lemma 16, then $\rho(F(\beta-\alpha)) \leq \max \{\rho(F), \rho(\alpha)\}$. Rewrite the function $F=(f-\alpha) /(\beta-\alpha)$ as $f=\alpha+F(\beta-\alpha)$. Thus, by Lemma 16, we conclude that $\rho(f) \leq \max \{\rho(\alpha), \rho(F(\beta-\alpha))\} \leq$ $\max \{\rho(\alpha), \rho(F)\}$. Furthermore, it follows from $\rho(\alpha)<\rho(f)$ that $\rho(f) \leq \rho(F) \leq \max \left\{q, q-\left(q_{2}-q_{1}\right) / k\right\}$.

If $\rho(\alpha)=\rho(f)$, noting that $\rho(\alpha)=\operatorname{deg} Q$, then $\rho(f)=$ $\rho(\alpha)=\operatorname{deg} Q=q \leq \max \left\{q, q-\left(q_{2}-q_{1}\right) / k\right\}$.

If $\rho(\alpha)>\rho(f)$, noting that $F=(f-\alpha) /(\beta-\alpha)=(f-$ $\left.Q_{1} e^{Q}\right) /\left(Q_{2}-Q_{1}\right) e^{Q}=f / P e^{Q}-Q_{1} / P$ and $Q_{1} / P$ is a rational function, then $\rho\left(Q_{1} / P\right)=0$ and $\rho(\alpha)=\operatorname{deg} Q=\rho\left(P e^{Q}\right)=$ $q>\rho(f)$. So, $\rho(f)<\operatorname{deg} Q=\rho(F) \leq \max \left\{q, q-\left(q_{2}-q_{1}\right) / k\right\}$.

Therefore, $\rho(f) \leq \rho(F) \leq \max \left\{q, q-\left(q_{2}-q_{1}\right) / k\right\}$ holds. This completes the proof of Theorem 6 .

\section{The Proof of Theorem 10}

Based on some ideas in [9], we will prove the theorem.

By Theorem 6, Lemma 17, and the assumption of Theorem 10 , we have

$$
\frac{f^{(k)}-\beta}{f-\beta}=\mu,
$$

where $\mu$ is a nonzero constant. It follows that

$$
f^{(k)}=\mu f+(1-\mu) Q_{2} e^{Q} .
$$

If $\mu=1$, then $f=f^{(k)}$; this is result (1) in Theorem B. Assuming that $\mu \neq 1$ and $z_{0}$ is a zero of $f-Q_{1} e^{Q}$, we have $f^{(k)}\left(z_{0}\right)-Q_{1}\left(z_{0}\right) e^{\mathrm{Q}\left(z_{0}\right)}=0$. Substituting $z_{0}$ into (59), we have

$$
Q_{2}\left(z_{0}\right)-Q_{1}\left(z_{0}\right)=0 .
$$

Noting that $Q_{2}-Q_{1} \neq 0$, we know that all the zeros of $f-Q_{1} e^{Q}$ are the zeros of $Q_{2}-Q_{1}$, so $f-Q_{1} e^{Q}$ has only finitely many zeros. Set $f=Q_{1} e^{Q}+P_{1} e^{Q_{\star}}$, where $P_{1}$ is a nonzero polynomial 
and $Q_{\star}$ is a polynomial. Then $f^{(k)}=P_{2} e^{Q}+\left(P_{1} Q_{\star}^{\prime k}+P_{3}\right) e^{Q_{\star}}$, where $P_{2}=\left(Q_{1} e^{Q}\right)^{(k)} / e^{Q}$ is a polynomial with $\operatorname{deg} P_{2}=$ $\operatorname{deg} Q_{1} Q^{\prime k}$ and $P_{3}$ is a polynomial with $\operatorname{deg} P_{3}<\operatorname{deg} P_{1} Q_{\star}^{\prime k}$. Substituting them into (59), we have

$$
\begin{aligned}
P_{2} e^{Q}+\left(P_{1} Q_{\star}^{\prime k}+P_{3}\right) e^{Q_{\star}} \\
\quad=\mu Q_{1} e^{Q}+\mu P_{1} e^{Q_{\star}}+(1-\mu) Q_{2} e^{Q} .
\end{aligned}
$$

Then,

$$
\begin{aligned}
P_{2} & =\mu Q_{1}+(1-\mu) Q_{2}, \\
P_{1}\left(Q_{\star}^{\prime}\right)^{k}+P_{3} & =\mu P_{1} .
\end{aligned}
$$

From (63), we get $Q_{\star}^{\prime k}=\mu$ and $P_{3}=0$. Thus $Q_{\star}^{\prime}$ is a constant, say $Q_{\star}^{\prime}=\lambda$. So $Q_{\star}(z)=\lambda z+b$ and $\mu=\lambda^{k}$, where $b$ is a constant. Furthermore,

$$
\begin{gathered}
f(z)=Q_{1}(z) e^{Q(z)}+P_{1}(z) e^{\lambda z+b} \\
f^{(k)}(z)=P_{2} e^{Q}+\left(P_{1} \lambda^{k}+P_{3}\right) e^{\lambda z+b}
\end{gathered}
$$

We deduce that $\operatorname{deg}\left(P_{3}\right)=\operatorname{deg}\left(P_{1}^{\prime}\right)$. Since $P_{3}=0$, we get that $P_{1}$ is a constant. Set $A=P_{1} e^{b}$ as a constant. Due to the fact that $\rho(\beta)<\rho(f)$ and (64), $Q(z)$ must be a constant. Hence, we complete the proof of Theorem 10 .

\section{Conflicts of Interest}

The authors declare that there are no conflicts of interest.

\section{Acknowledgments}

This work was supported by NNSF of China (11501367 and 1701111), NSF of Guangdong Province (2016A030310257), and Leading Academic Discipline Project of Shanghai Dianji University (16JCXK02).

\section{References}

[1] H. X. Yi and C. C. Yang, Uniqueness Theory of Meromorphic Functions, Science Press, Beijing, China, 1995.

[2] L. A. Rubel and C. C. Yang, "Values shared by an entire function and its derivative," in Complex Analysis, vol. 599 of Lecture Notes in Math, pp. 101-103, Springer, Berlin, Germany, 1977.

[3] F. Lü, J. Xu, and A. Chen, "Entire functions sharing polynomials with their first derivatives," Archiv der Mathematik, vol. 92, no. 6, pp. 593-601, 2009.

[4] X.-M. Li and C.-C. Gao, "Entire functions sharing one polynomial with their derivatives," The Proceedings of the Indian Academy of Sciences - Mathematical Sciences, vol. 118, no. 1, pp. 13-26, 2008.

[5] R. Gu, Z. Li, and W. Yuan, "The growth of entire solutions of some algebraic differential equations," Georgian Mathematical Journal, vol. 18, no. 3, pp. 489-495, 2011.

[6] R. Halburd and R. Korhonen, "Value distribution and linear operators," Proceedings of the Edinburgh Mathematical Society, vol. 57, no. 2, pp. 493-504, 2014.
[7] R. Korhonen, "An extension of Picard's theorem for meromorphic functions of small hyper-order," Journal of Mathematical Analysis and Applications, vol. 357, no. 1, pp. 244-253, 2009.

[8] G. Zhang and J. Wang, "The infinite growth of solutions of complex differential equations of which coefficient with dynamical property," Taiwanese Journal of Mathematics, vol. 18, no. 4, pp. 1063-1069, 2014.

[9] F. Lü and H. Yi, "The Brück conjecture and entire functions sharing polynomials with their $k$-th derivatives," Journal of the Korean Mathematical Society, vol. 48, no. 3, pp. 499-512, 2011.

[10] J. L. Schiff, Normal Families, Universitext, Springer, Berlin, Germany, 1993.

[11] X. Pang and L. Zalcman, "Normal families and shared values," Bulletin of the London Mathematical Society, vol. 32, no. 3, pp. 325-331, 2000

[12] F. Lü and J. Qi, "A note on the hyper-order of entire functions," Bulletin of the Korean Mathematical Society, vol. 50, no. 4, pp. 1209-1219, 2013.

[13] J. Zhang and L.-W. Liao, “On Brück's conjecture on entire functions sharing one value with their derivatives," Houston Journal of Mathematics, vol. 36, no. 2, pp. 665-674, 2010.

[14] J. Grahl and C. Meng, "Entire functions sharing a polynomial with their derivatives and normal families," Analysis International Mathematical Journal of Analysis and its Applications, vol. 28, no. 1, pp. 51-61, 2008.

[15] P. Yang and S. Nevo, "Derivatives of meromorphic functions with multiple zeros and elliptic functions," Acta Mathematica Sinica, vol. 29, no. 7, pp. 1257-1278, 2013.

[16] P. Yang, "Problems on $\left(f^{2}\right)^{(k)}$," Journal of Mathematical Analysis and Applications, vol. 335, no. 2, pp. 902-906, 2007. 


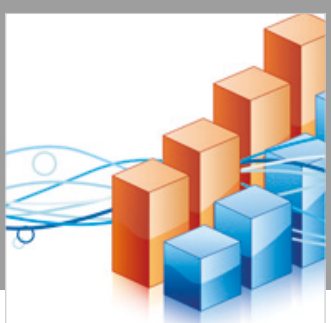

Advances in

Operations Research

\section{-n-m}
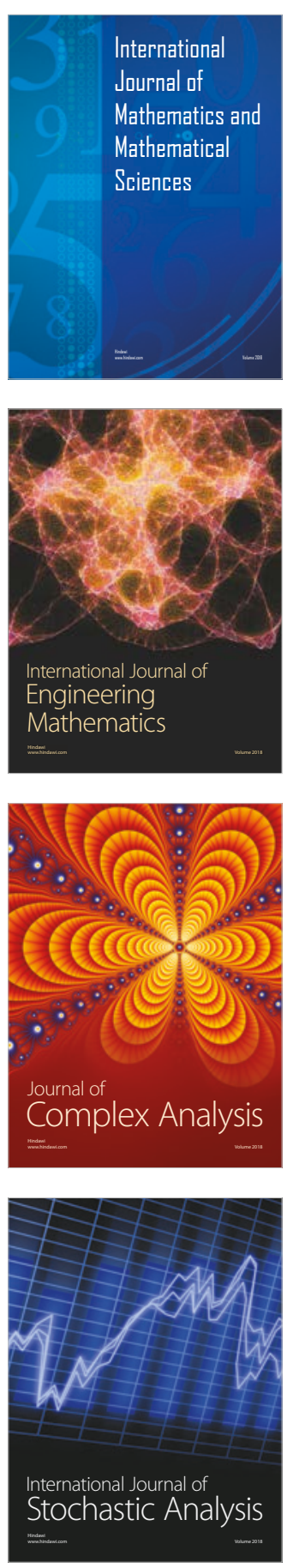
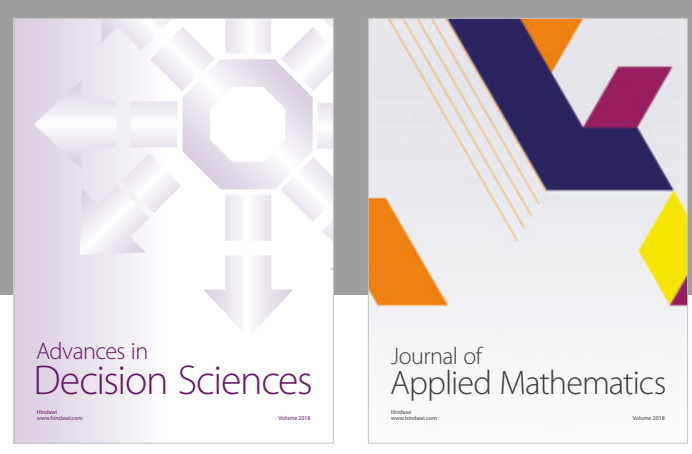

Journal of

Applied Mathematics
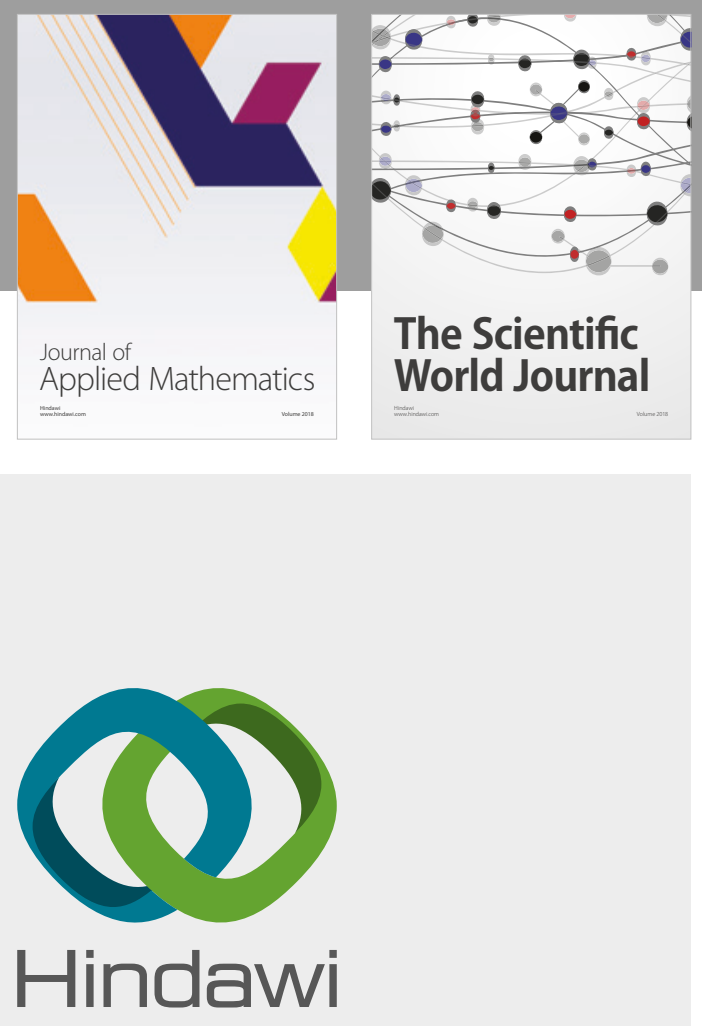

Submit your manuscripts at

www.hindawi.com

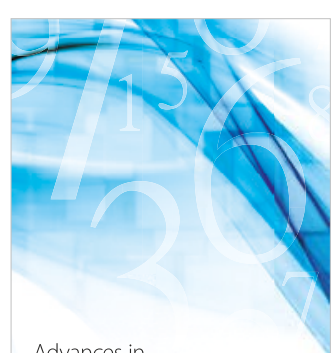

Advances in
Numerical Analysis
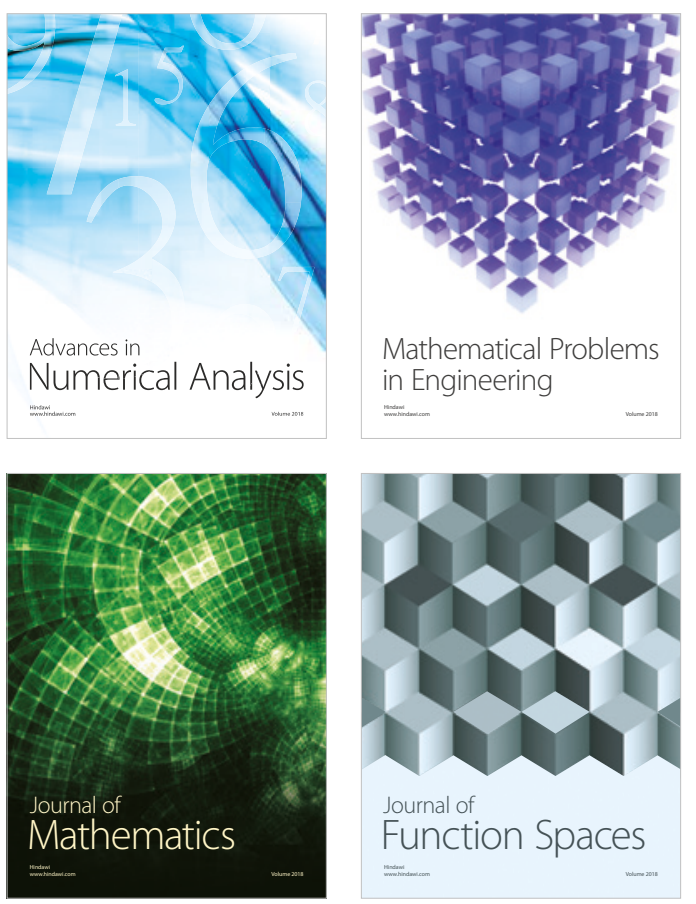

Mathematical Problems in Engineering

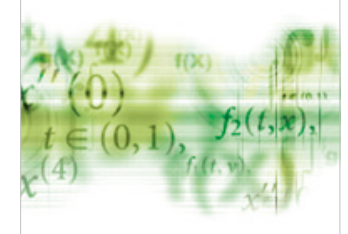

International Journal of

Differential Equations

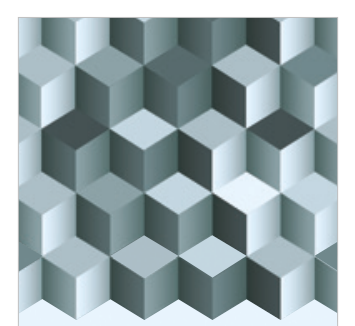

Journal of

Function Spaces

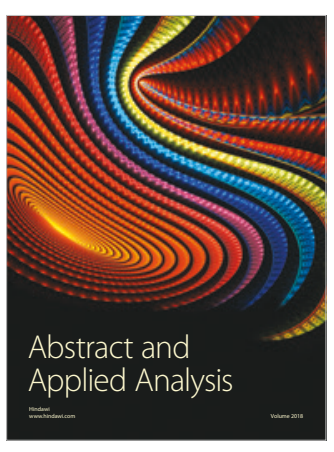

The Scientific

World Journal

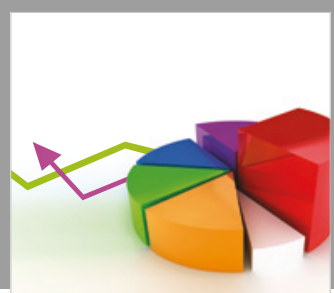

Journal of

Probability and Statistics
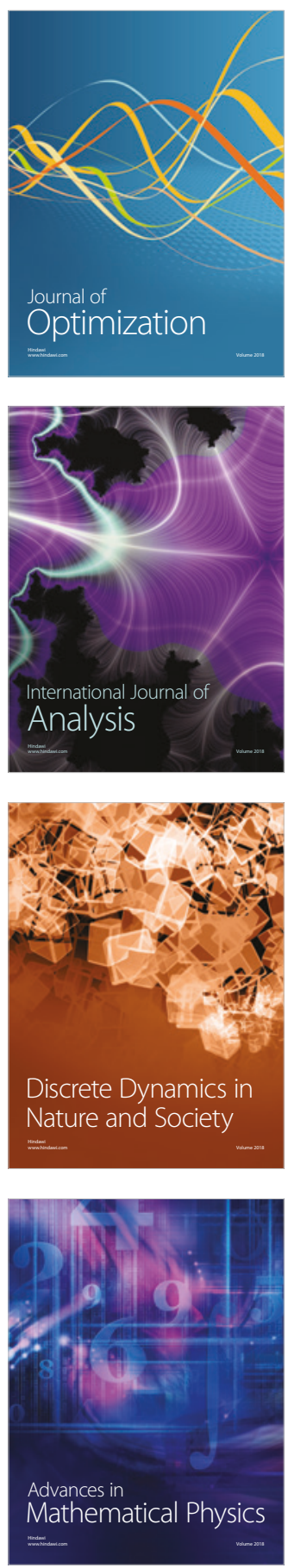